\title{
NRT2.4 and NRT2.5 Are Two Half-Size Transporters from the Chlamydomonas NRT2 Family
}

\author{
Jose Javier Higuera ${ }^{1,+}$, Victoria Calatrava ${ }^{1,+}{ }^{,}$Zaira González ${ }^{1}$, Vicente Mariscal ${ }^{2}$, \\ Jose Manuel Siverio ${ }^{3}$, Emilio Fernández ${ }^{1}$ and Aurora Galván ${ }^{1, *}$ \\ 1 Departamento de Bioquímica y Biología Molecular, Facultad de Ciencias, Universidad de Córdoba, \\ Campus de Rabanales, Campus de excelencia internacional (CeiA3), Edif. Severo Ochoa, \\ Córdoba E-14071, Spain; b92hisoj@uco.es (J.J.H); b82capom@uco.es (V.C.); \\ zgonzalezsanchez@gmail.com (Z.G); bb1feree@uco.es (E.F.) \\ 2 Instituto de Bioquímica Vegetal y Fotosíntesis, Consejo Superior de Investigaciones Científicas and \\ Universidad de Sevilla, Américo Vespucio 49, Sevilla E-41092, Spain; vicente.mariscal@ibvf.csic.es \\ 3 Departamento de Bioquímica y Biología Molecular, Grupo del Metabolismo del Nitrógeno, \\ Universidad de La Laguna, La Laguna E-38206, Tenerife, Spain; jsiverio@ull.es \\ * Correspondence: bb1gacea@uco.es; Tel./Fax: +34-957-218-591 \\ + These authors contributed equally to this work
}

Academic Editors: Anne Krapp and Bertrand Hirel Received: 31 October 2015; Accepted: 14 March 2016; Published: 19 March 2016

\begin{abstract}
The NRT2 transporters mediate High Affinity Nitrate/NitriteTransport (HAN/NiT), which are essential for nitrogen acquisition from these inorganic forms. The NRT2 proteins are encoded by a multigene family in plants, and contain 12 transmembrane-spanning domains. Chlamydomonas reinhardtii has six NRT2, two of which (NRT2.5 and NRT2.4) are located in Chromosome III, in tandem head to tail. cDNAs for these genes were isolated and their sequence revealed that they correspond to half-size NRT2 transporters each containing six transmembrane domains. NRT2.5 has long $N$ - and C- termini sequences without known homology. NRT2.4 also contains long termini sequences but smaller than NRT2.5. Expression of both studied genes occurred at a very low level, slightly in darkness, and was not modified by the $\mathrm{N}$ or $\mathrm{C}$ source. Silencing of NRT2.4 by specific artificial miRNA resulted in the inhibition of nitrite transport in the absence of other HANNiT (NRT2.1/NAR2) in the cell genetic background. Nitrite transport activity in the Hansenula polymorpha $\triangle y n t:: U R A 3$ Leu2 mutant was restored by expressing CrNRT2.4. These results indicate that half-size NRT2 transporters are present in photosynthetic organisms and that NRT2.4 is a HANiT.
\end{abstract}

Keywords: half-size transporter; nitrite transport; gene expression; Hansenula complementation

\section{Introduction}

Nitrate is an essential mineral nutrient for plant growth and development. In fact, nitrate is reduced to ammonium and used to build biological molecules such as amino acids, proteins or nucleic acids. It is also a signal molecule that regulates the expression of genes for nitrate assimilation, root architecture, and other metabolic processes in the plant [1-3]. The nitrate concentrations in plant soils might change extremely from $10 \mu \mathrm{M}$ in natural soils to $100 \mathrm{mM}$ in fertilized soils [1]. Plants have displayed a sophisticated network of membrane transporters for the sensing, absorption, storage, and distribution of nitrate inside the organism. These nitrate transporters belong to four families: NRT1/PTR/NPF (nitrate transporter 1/peptide transporter/nitrate peptide transporter family), NRT2, chloride channel (CLC), and slow anion channel-associated 1 homolog 3 (SLAC1/SLAH), which have been reviewed $[4,5]$. 
Nitrate is also a nutrient for algae, which share many aspects for nitrate assimilation and regulation with land plants [6-8]. Water is the natural habitat for algae, where nitrate concentration is usually less than $1 \mu \mathrm{M}$ and could increase several orders of magnitude due to contamination from plant fertilizers. The unicellular and model alga Chlamydomonas reinhardtii also has a high number of transporters to control cellular nitrate/nitrite and for adapting it to the changing environmental conditions, but this is still not fully understood. Thus, Chlamydomonas is a powerful green model that can provide more detailed analysis of the molecular function of genes for nitrate and nitrite transport studies [6-8]. This knowledge is key for understanding how the plants use nitrate fertilizers, one important topic in agronomy. In Chlamydomonas, the nitrate/nitrite transporters belong to the NPF and NRT2 as in plants, but this alga also has NAR1 transporters that are absent in plant genomes. NAR1 belongs to the formiate/nitrite transporter family (FNT) present in bacteria (FocA and NirC, in E. coli), fungi (NitA in Aspergillus nidulans), and yeast (NAR1 in Hansenula polymorpha). The structure of FNT is different from NPF and NRT2 transporters. FNTs are six transmembrane helixes proteins that can form a pentameric structure similar to aquaporin and behave more like a channel than a transporter [9]. The functions for the different NAR1/FNT can be summarized as: (1) in E. coli, NirC is involved in both nitrite uptake and export [10]; (2) in Aspergillus nidulans, NitA mediates specific high-affinity transport of nitrite and also has some role in nitrite efflux [11]; (3) in Hansenula, NAR1 mediates nitrate and nitrite efflux [12]; (4) in Chlamydomonas reinhardtii there exist six NAR1 and their functions have only been studied for NAR1.1 and NAR1.2. NAR1.1 is a plastidic nitrite transporter and, together with other key proteins for nitrate assimilation, is nitrate regulated and under the control of the major regulatory gene NIT2 [13]. NAR1.2, also named LCIA, is a plastidic transporter with specificity for both nitrite and bicarbonate, and overexpressed under low $\mathrm{CO}_{2}$ conditions [14]. Recently, it has been shown that NAR1.2 (LCIA) is a chloroplast envelope $\mathrm{HCO}_{3}{ }^{-}$transporter that forms part of a $\mathrm{CO}_{2}$ concentrating mechanism (CCM) operating at very low $\mathrm{CO}_{2}[15,16]$. The $\mathrm{CCM}$ is essential in aquatic microalgae to accumulate $\mathrm{CO}_{2}$ close to RUBISCO and to make efficient photosynthesis. More details for nitrate assimilation in microalgae have been recently reviewed [8].

The high-affinity nitrate transporters NRT2 are present in plants, algae, fungi, yeast, and bacteria [17-23]. This transporters family is also named NNP (Nitrate Nitrite Porter) (TC 2.A.1.8) and belongs to the Major Facilitator Superfamily (MFS) (TC 2.A.1) [24,25]. The structure of a typical NRT2 protein consists of 12 transmembrane helixes in two sets of six that form an $N$-terminal domain and a C-terminal domain linked by a hydrophilic central loop between helixes 6 and 7 [26]. This central loop is long, more than 90 residues, in fungal and yeast NRT2. The sequence identity between NRT2 proteins goes from $30 \%$ to $90 \%$, but the highest conservation is in the middle of the protein where the MFS and NNP motifs are identified $[18,24,26]$. Meanwhile, the Aspergillus NRTA and NRTB, the Hansenula YNT, and the E. coli NARK and NARU do not require a second component for functionality; most of the NRT2 from plants and some from Chlamydomonas requires a second protein NAR2 [27-32]. In Arabidopsis, the two-components system NRT2/NAR2 is proposed to function as a tetrameric complex formed by two subunits from each of the two polypeptides [32,33]. All NRT2 studied are high-affinity transporters; in plants they are shown to work as high affinity nitrate transporters [32], whereas in fungi and yeast (AnNRTA, AnNRTB, HpYNT), as well as in Chlamydomonas (NRT2.1/NAR2), they are high affinity nitrate/nitrite transporters $[11,22,34,35]$.

Chlamydomonas genome has six NRT2 genes [8]. Two of them (NRT2.4 and NRT2.5) are predicted to encode atypical NRT2 proteins in terms that they are half-size molecules. These genes have been analyzed in the present work and it is shown that NRT2.4 is a high affinity nitrite transporter. 


\section{Results}

\subsection{Half-Size Transporters Encoded by NRT2.4 and NRT2.5}

Chlamydomonas genome predicts two NRT2 genes in chromosome III that encode putative NRT2 transporters with six transmembrane helixes. Those transporter genes, named NRT2.5 and NRT2.4, are in orientation from tail to head. The putative proteins contain elements from the first and second half of the typical twelve-transmembrane NRT2/NNP proteins. The isolation of the corresponding cDNAs was achieved according to the predictions from Chlamydomonas jgi v2 (Figure 1). Two amplification rounds with nested primers were performed, first with 5'UTR2.5-Qo and second with ATG2.5-Qi, and resulted in a 3-kb band, which was cloned and sequenced (Figure 1AB). The nucleotide sequence showed a polyadenylated transcript corresponding to NRT2.5 where a canonical TGTAA polyadenylation signal was present $15 \mathrm{nt}$ before the polyA tail, and the $3^{\prime} \mathrm{UTR}$ sequence, $171 \mathrm{nt}$-length, coincided with this region in the genome. The existence of the polyadenylated NRT2.5 was confirmed in three Chlamydomonas strains: two wild types for nitrate assimilation 704 and 6145c, and the mutant D2 (Figure 1C). The sequencing data confirmed the existence of a NRT2.5 transcript from different strains and support the presence of an atypical half-size NRT2 transporter in Chlamydomonas. To check whether a second half-size NRT2.4 transporter is also expressed, an RT-PCR amplification of the corresponding ORF was achieved (Figure 1D). Also, nested primers were used, the first round with 5'UTR_Nrt2.4-LoSpeI_Nrt2.4 and second round with ATG_Nrt2.4-LoSpeI_Nrt2.4. The amplified $1.5 \mathrm{~Kb}$ band (Figure 1D) was cloned, and its sequence corresponded to a cDNA for NRT2.4. Thus, the sequence analysis shows the existence of two independent cDNAs corresponding to NRT2.5 and NRT2.4 for which their exon/intron organization is shown in Figure 1, and the encoded proteins correspond to half-size NRT2 transporters (Figure 2).

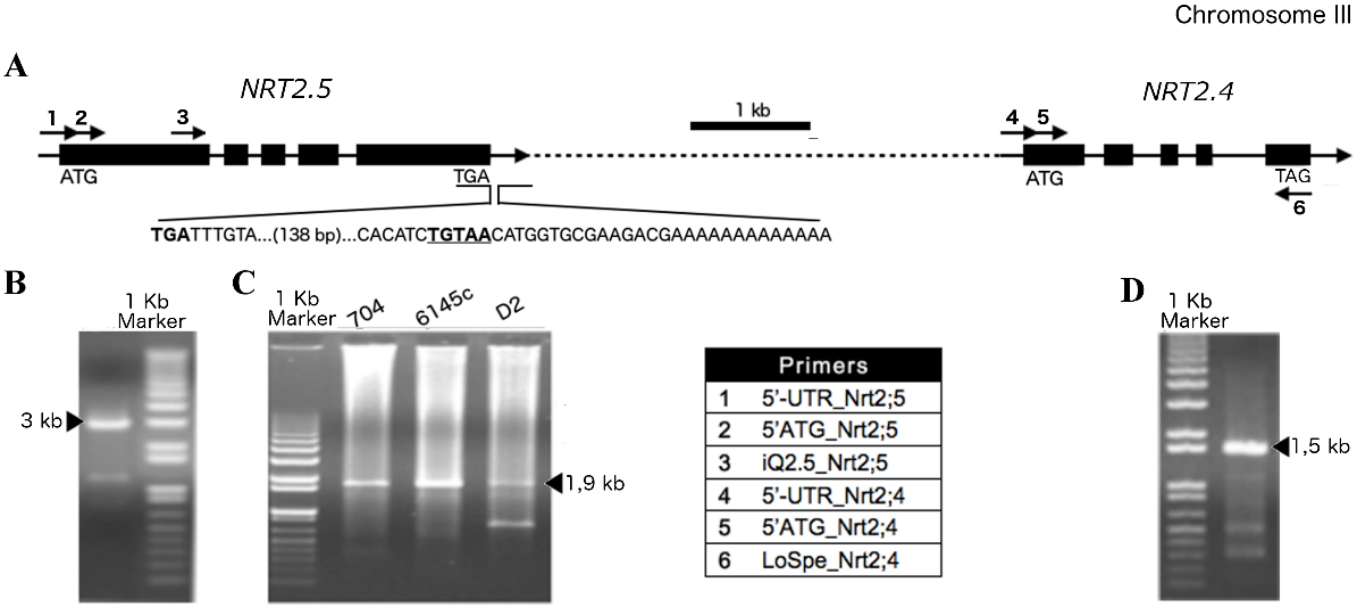

Figure 1. Amplification of cDNA corresponding to NRT2.5 and NRT2.4. (A) Scheme of the genomic region containing NRT2.4 and NRT2.5, where the exon/intron organization and the localization of primers used to amplify the corresponding cDNAs are indicated (B) Amplification of NRT2.5 cDNA with primers 1 and $Q_{0}$ in a first lap, and primers 2 and $Q_{i}$ in a following lap. The 3-kb band, corresponding to full cDNA of NRT2.5 was cloned and sequenced; (C) Probing the existence of polyadeylated NRT2.5 in three Chlamydomonas strains, the WT strains 704 and 6145c, and in D2 strain (deleted at NRT2.1, NRT2.2, NAR2). RT-PCR was performed with primers 3 and $\mathrm{Q}_{0}$ in a first lap, and primers 3 and $Q_{i}$ in a following lap. The amplified $1.9 \mathrm{~kb}$ band was sequenced; (D) Amplification of the ORF corresponding to NRT2.4. The ORF of NRT2.4, a $1.5 \mathrm{~kb}$ band, was amplified with primers 4 and 6 in a first lap, and 5 and 6 in a second lap. In B and D, cDNA from the strain 704 was used. 


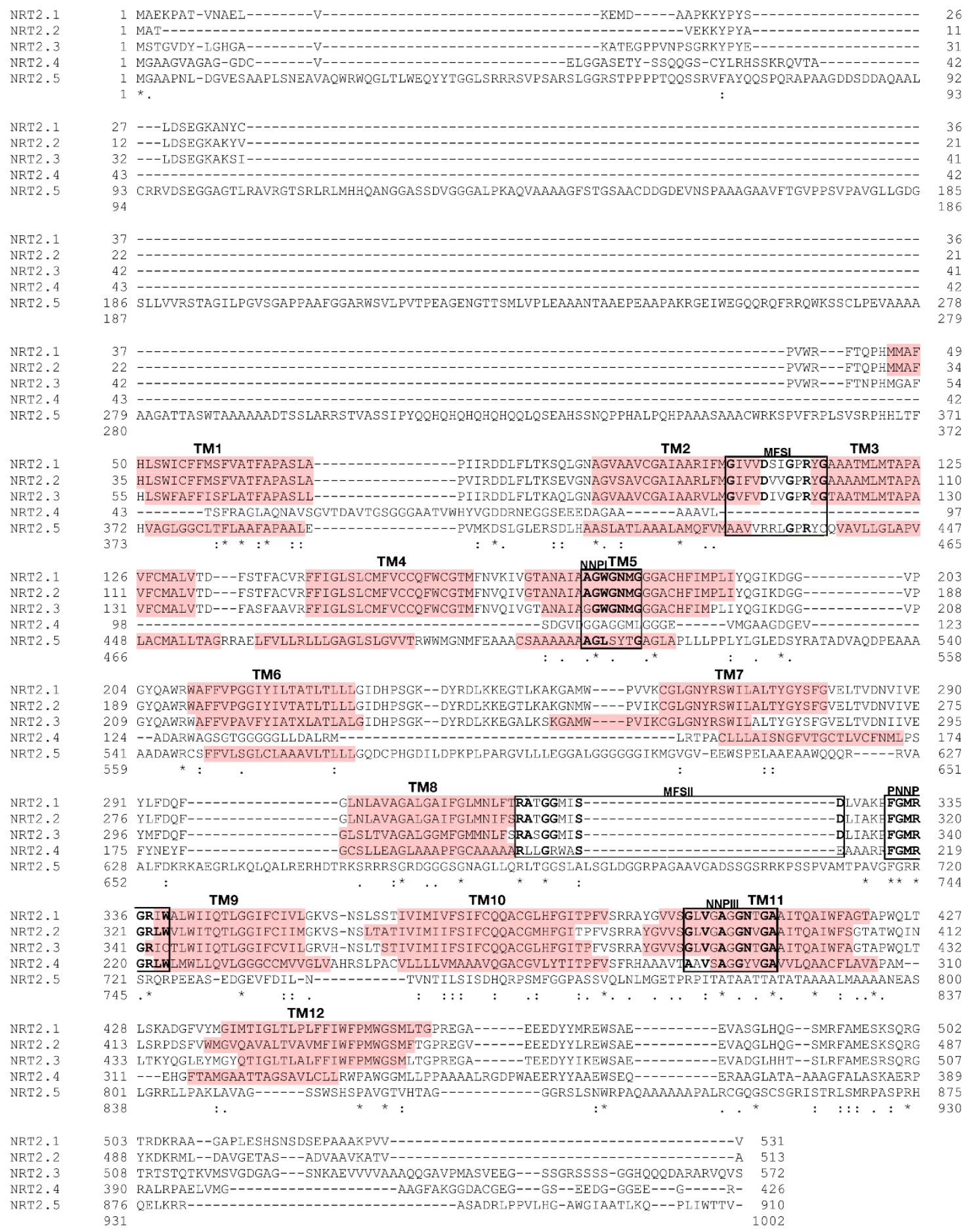

Figure 2. Alignment of NRT2 protein sequences from C. reinhardtii. This alignment was performed using TM-Coffee. Dashes refer to expansion of the sequence to allow maximal alignment. Sequences of transmembrane regions are shadowed and named TM1-12. Boxes indicate conserved signatures, MFSI, NNPI, MFSII, NNPII, and the photosynthetic NNP (PNNP) signature FGMRGR. Protein identification corresponds to: NRT2.1 (Cre09.g410850), NRT2.2 (Cre09.g410800), from Phytozome 10.3; NRT2.3 (AY669386.1) accession number in NCBI, NRT2.4 (KT971135), and NRT2.5 (KT971134). The prediction of transmembrane helices of each protein was obtained using the HMMTOP program [36]. 
These NRT2 proteins, 910 amino acids for NRT2.5 and 426 amino acids for NRT2.4, show homology with NRT2 transporters (Figure 2). The comparison of the Chlamydomonas NRT2.1-5 (Supplementary Table S1A) was performed by comparing one on one each of the two halves of NRT2.1-3 (from transmembrane domains 1 to 6 , and 7 to 12) and NRT2.4 and 5. As known, there is a high identity between NRT2.1 and $2(86 \%-94 \%)$ and between NRT2.2 and 2.3 about $86 \%$. The identity degree between NRT2.4 and NRT2.1-3 is around 35\%-37\% and is similar between NRT2.5 and NRT2.1-3. The least identity is shared between NRT2.4 and NRT2.5. Comparisons of the Chlamydomonas full NRT2.1-5 proteins (Supplementary Table S1B) gave similar results, though with a smaller degree of conservation than above.

In addition, particular features were observed when analyzing NRT2.5 and NRT2.4 together with NRT2.1-3 transporters (Figures 2 and 3). The NRT2.5 transporters (910 residues) adjust to a six transmembrane protein with conservation with the first part of a typical NRT2 protein. NRT2.5 contains a MSF-I motif, between the second a third transmembrane domains, and a NNP-I motif into the fifth transmembrane helix. The expanding $\mathrm{N}$-terminus (370 residues) and C-terminus ( 342 residues) have no homology with NRT2 proteins. This is usual within the NRT2/NNP family in which the middle region of the proteins shares a high degree of similarity, whereas the $\mathrm{N}$ - and C-termini share low homology [18].

The NRT2.4 protein (426 residues) has also six transmembrane helixes, which fits to the second part of a typical NRT2 protein (Figure 3). Thus, NRT2.4 contains a MSF-II motif between its second and third helix, which corresponds to the region between the eight and nine helixes in a typical NRT2. NRT2.4 also contains the NNP-II motif in its fifth transmembrane helix, which corresponds to the region in transmembrane 11 in a typical NRT2 protein. In addition, NRT2.4 contains a conserved motif FGMRGRLW considered as characteristic of eukaryotic photosynthetic NRT2 proteins [18]. The $N$-terminus (149 residues) and C-terminus (89 residues) sequences of NRT2.4, smaller than in NRT2.5, have no homology with NRT2 proteins.

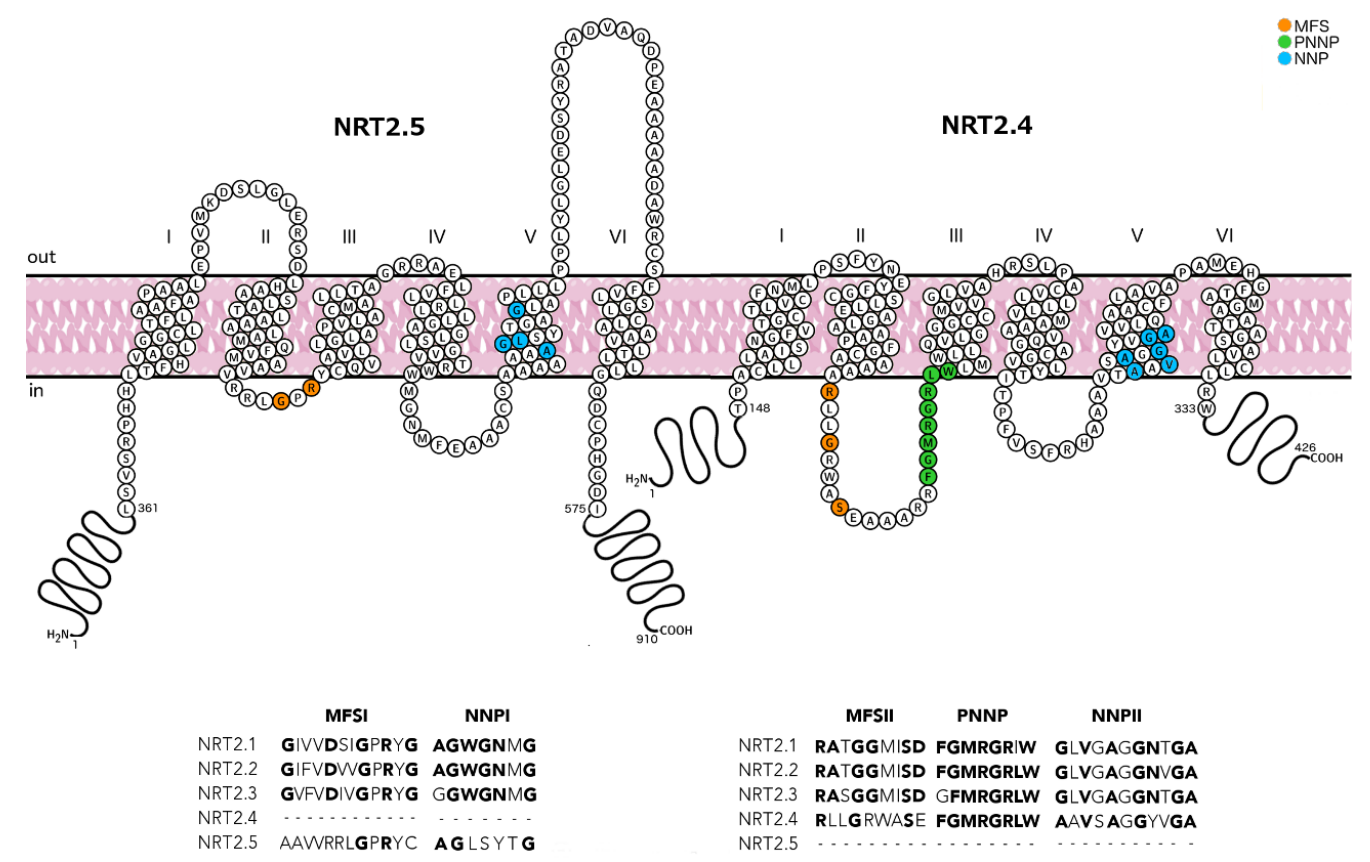

Figure 3. Predicted membrane topology for NRT2.4 and NRT2.5 from Chlamydomonas. The model was obtained by using the PROTTER program [37]. Strictly conserved amino acids corresponding to each motif signature are colored in orange for the Major Facilitator Superfamily motif (MFSI and II), blue for Nitrate Nitrite Porter motif (NNPI and II), and green for the Photosynthetic Nitrate Nitrite Porter motif (PNNP). 


\subsection{Expression of NRT2.4 and NRT2.5 is Not Regulated by Nitrogen}

The main characteristics of the Chlamydomonas NRT2.1, NRT2.2, and NRT2.3 expression are its up-regulation by nitrate, and that they are under the control of NIT2, the master gene for nitrate assimilation in the alga [7,38]. The expression of NRT2.4 and NRT2.5 genes has been analyzed under different conditions: high versus low $\mathrm{CO}_{2}$, ammonium versus nitrate, and light versus darkness (Figure 4). The results show that both genes have very low expression levels and that they are not regulated by nitrogen or carbon, but in the dark their expression seems to be slightly increased.
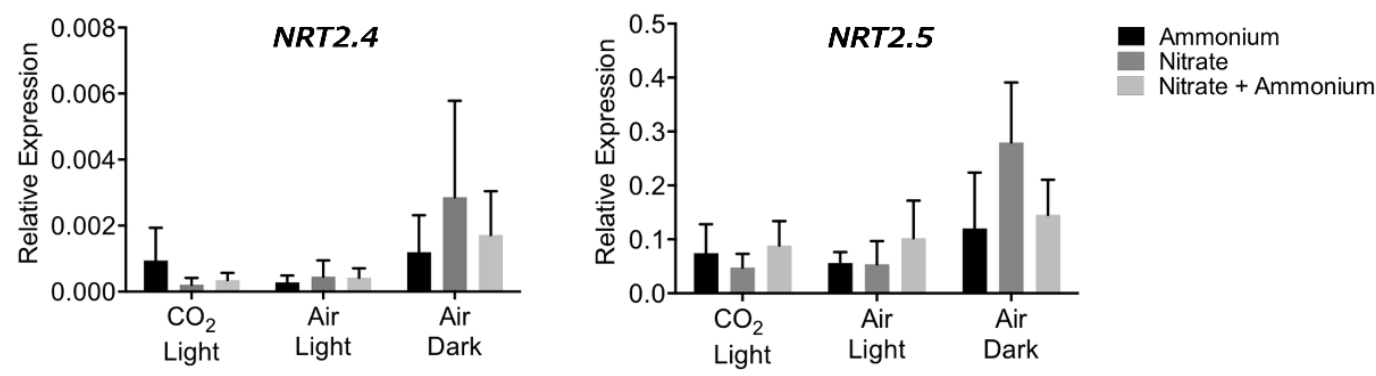

Figure 4. Expression of NRT2.4 and NRT2.5. Ammonium-grown cells were transferred to medium containing $4 \mathrm{mM}$ of the indicated nitrogen sources, in the presence of light or in the dark, and with or without $\mathrm{CO}_{2}(5 \%)$. After three hours, samples were processed for real-time quantification by using specific primers indicated in Supplementary Table S2. Gene transcript levels are given as relative abundance with respect to the housekeeping gene ubiquitin ligase. The Chlamydomonas strain used is the WT strain 21gr.

\subsection{NRT2.4 is a Nitrite Transporter}

Although NRT2.5 and NRT2.4 are half-size of a typical NRT2, they could work as an NNP transporter: (1) by interaction of NRT2.5 (first part) and NRT2.4 (second part) to give a complete NRT2 protein; or (2) just as a half-sized NRT2.

An artificial microRNA technique has been used to silence NRT2.4 [39]. The construction was cloned in a vector pChlamiRNA3 that confers paromomycin resistance, and was used for selection of the Chlamydomonas transformants (see Experimental Section). Three Chlamydomonas strains with different genetic backgrounds for NRT2 transporters were transformed with the amiRNA construction. Strain 704 is a wild type, JA4 (contains NRT2.1/NAR2 and 2.3 and lacks NRT2.2), and finally strain D2R4 (which has NRT2.3 and lacks NRT2.1, NRT2.2 and NAR2). Due to the low expression level of NRT2.4, the determination of the magnitude of silencing for this gene expression in the isolated transformants was subjected to a very high error. Different isolated paromomycin-resistant strains were used for the analysis of nitrite transport activity (Figure 5). The nitrite transport activity from the above strains and transformants under two conditions is shown. These cells were grown in an ammonium medium and nitrite transport activity was induced either directly (Figure 5A) or after induction of the cells in $4 \mathrm{mM}$ nitrate for three hours (Figure 5B). No significant difference on this transport activity was observed between those strains. In Figure $5 C, D$, the nitrite transport activity from the strain JA4 and a transformant was compared under the same conditions as above and no significant differences were obtained between them as well. When NRT2.4 was silenced under a D2R4 genetic background (five strains randomly isolated), after nitrate induction, transformants showed a lower nitrite transport activity than their parental strain (Figure 5E). The activity of the nitrite transport was from $100 \%$ in D2R4 to $14 \%-72 \%$ in D2AS strains. It is worth noting that to detect nitrite transport activity from the $\mathrm{D} 2$ strains and under the assayed conditions, high $\mathrm{CO}_{2}$, nitrate pre-incubation is required to induce nitrite transport [38]. 
A

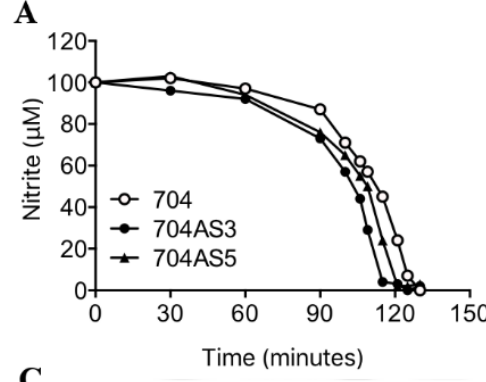

C

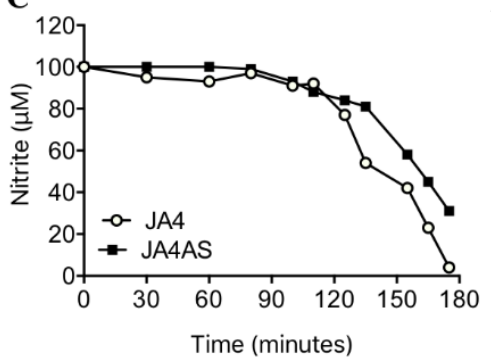

B

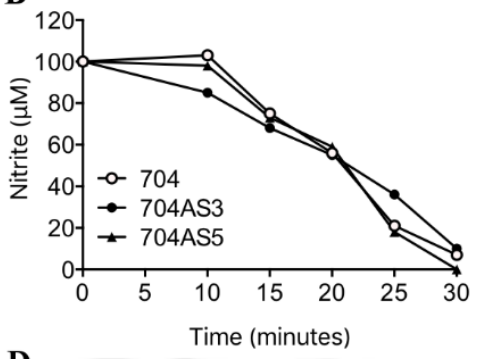

D

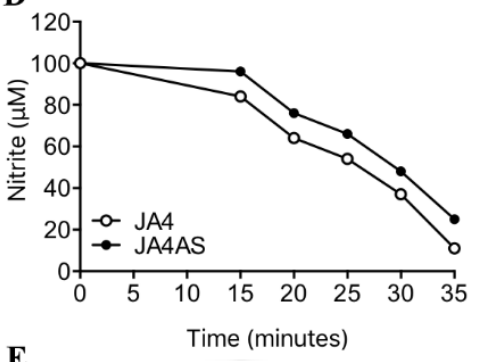

$\mathbf{E}$

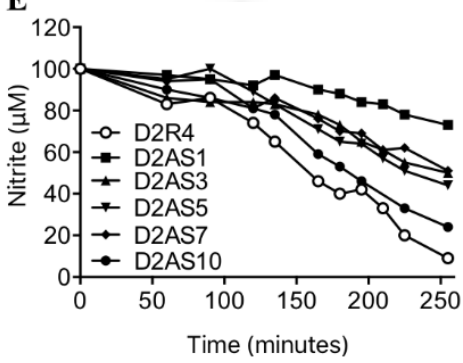

Figure 5. Nitrite uptake activity by Chlamydomonas strains when NRT2.4 is silenced. NRT2.4 silencing was performed by the artificial miRNA technique (see Experimental Section). Strains correspond to a wild type 704 and its corresponding silencing transformants (ami, 704AS3 and 704 AS5), JA-4 strain that contains NRT2.1 and NAR2 but lacks NRT2.2, as well as its corresponding ami (JA4S), and D2R4 that lacks of NRT2.1, NRT2.2 and NAR2, as well as its corresponding ami (D2R4AS1, AS3, AS5, AS7 and AS10). (A) and (C), ammonium-grown cells were washed and transferred to $100 \mu \mathrm{M}$ of nitrite. (B), (D) and (E) cells were previously induced in $4 \mathrm{mM}$ nitrate medium during three hours and then transferred to $100 \mu \mathrm{M}$ nitrite. The nitrite concentration in the medium was quantified along with time. Cells were always bubbled with $5 \% \mathrm{CO}_{2}$ enriched air. Cell density of the cultures was adjusted to $10 \mathrm{mg} \mathrm{Chl} / \mathrm{L}$. Data correspond to the average of a single replicate.

In Chlamydomonas, NRT2.1/NAR2 is the most efficient high affinity transporter and bispecific for nitrate and nitrite [6,7]. Thus, data from Figure 5 suggest that NRT2.4 participates in nitrite transport, but its activity is masked by NRT2.1/NAR2.

To determine whether this NRT2.4, half-size NRT2, is sufficient to mediate nitrite transport activity, its expression in yeast was assayed. The ORF for NRT2.4 was cloned into the yeast pYNR vector and used to complement the Hansenula polymorpha $\triangle y n t:: U R A 3$ Leu2 mutant. This mutant has no YNT1, the only NRT2 transporter described in this yeast, and is affected in nitrate and nitrite transport [40]. Three yeast transformants expressing the Chlamydomonas NRT2.4 ( $\triangle y$ ntcrNRT2.4 L1-3) were assayed for nitrite transport activity under the conditions previously described [40]. Yeast cells were induced for $90 \mathrm{~min}$ in YG medium without nitrogen, and then nitrite transport activity was measured at $\mathrm{pH} 6.5$ and using $100 \mu \mathrm{M}$ nitrite. As shown in Figure 6, the $\Delta$ ynt mutant does not show nitrite transport activity, but the complementation with CrNRT2.4 restores this activity, though it was less efficient than in the Hansenula wild type. 


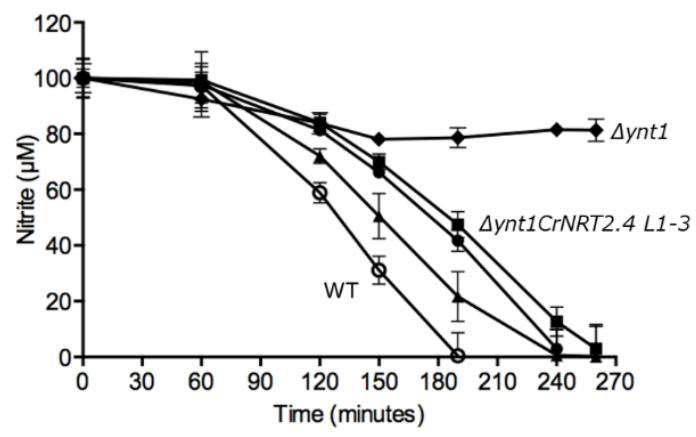

Figure 6. Nitrite uptake activity in Hansenula polymorpha expressing CrNRT2.4. The Hansenula strains correspond to WT strain (open circles), $\Delta y n t 1$ mutant (closed diamonds) and three CrNRT2.4:: $\Delta y n t 1$ transformants (L1-3) (closed circles, squares, and triangles, respectively). Nitrite uptake was performed at $\mathrm{pH} 6.5$ from cells induced for $90 \mathrm{~min}$ in minimal medium without nitrogen [40]. Cell density was adjusted at $660 \mathrm{~nm}$ and corresponded to $10 \mathrm{mg} / \mathrm{mL}$ (fresh weight).

\section{Discussion}

The genome of Chlamydomonas reinhardtii predicts six NRT2 genes [8,41,42]. Three of them, NRT2.1 to NRT2.3, have been previously characterized $[21,34,38,43]$ and correspond to the typical NRT2/NNP transporters with 12 transmembrane helixes. NRT2.1 together with NAR2 form a two-component high affinity system I, which is bispecific for nitrate and nitrite. NRT2.2 together with NAR2 form a high affinity system II, which is more specific for nitrate. The lack of NRT2.1, NRT2.2, and NAR2 genes results in the absence of high affinity nitrate transport activity under high $\mathrm{CO}_{2}$ in Chlamydomonas cells—which still have high affinity nitrite uptake activity, called system III-so it is NAR2 independent and was attributed to NRT2.3 [38]. A chlorate-resistant mutant isolated in the $\Delta$ [NRT2.1, NRT2.2, NAR2] background (strain DC2-III) resulted in the non- expression of this system III transport activity and the gene affected was found to be closely linked to NIT2 [38]. Later on, in DC2-III, a mobile element was identified within NIT2 at the end of NIT2 causing its inactivation [44]. The activity of another transport system named IV was also proposed in Chlamydomonas, which has the following characteristics: (1) it is detected under low $\mathrm{CO}_{2}$ conditions; (2) it has high-affinity for both nitrate and nitrite; and (3) it is inhibited by chloride and $\mathrm{CO}_{2}$ but not by ammonium [38,45]. In summary, Chlamydomonas seems to take up nitrite efficiently from the medium by systems that are independent of NRT2.1, NRT2.2, and NAR2.

The typical NRT2 protein has 12 transmembrane helixes that form two domains, one N-terminal domain (TM1-TM6) and one C-terminal domain (TM7-TM12), where MFS and NNP motifs are identified $[18,24,26]$. In transmembrane five a conserved signature motif NNP I (A-G-W/L-G-N-M-G) is present. A second NNP signature is found in transmembrane 11 and is related to the sequence F/Y/K-x3-I/L/Q/R/K-x-G/A-x-V/A/S/K-x-G/A/S/N-L/I/V/F/Q-x 1,2-G-x-G-N/I/M-x-G-G/V/T/A. Another conserved sequence, R-[PA]-x-G-G-x-x-[SA]-D, is identified between transmembranes 8 and 9 and considered MFS II motif. Half of this sequence (G-x-x-[SA]-D) is closely related to a part of the MFS specific sequence motif, G-[RKPATY]-L-[GAS]-[DN]-[RK]-[FY]-G-R-[RK]-[RKP]-[LIVGST]-[LIM], which is located between transmembranes 2 and 3. However, the longest conserved sequence, F-G-M-R-G-R-L-W, is at the beginning of transmembrane 9 and found in photosynthetic organisms [18].

In this work, it is shown that NRT2.4 and NRT2.5 encode atypical NRT2 proteins that are half-size of a 12 transmembrane NRT2. These genes are in chromosome III in orientation NRT2.5 $\rightarrow$ NRT2.4 and correspond to the N- and C-termini domains of a full NRT2. The identity degree between whole proteins goes from $28 \%$ (NRT2.5 versus NRT2.1) up to 31\% (NRT2.4 versus NRT2.1). However, in considering half molecules the identity degree increases up to $37 \%$ (NRT2.5 versus $N$-terminal of NRT2.1 or NRT2.4 versus C-terminal of NRT2.1). 
Up to now, this is the first report of half-size NNP transporters. The 12 transmembrane helixes are the most frequent topology of MFS transporters and believed to be the result of an ancient gene duplication of a gene encoding a single domain with six transmembrane helixes. The maintenance of both domains in a single polypeptide chain could perhaps have been favored during evolution in order to facilitate substrate recognition and conformational changes during the transport [25]. It has been reported that halves of sucrose transporters StSUT1 from Solanum tuberosum show intermolecular interactions and reconstitute sucrose transport activity similar to the intact protein [46]. Also, heteromeric complementation is obtained with $\mathrm{N}$-terminal halves from Lycopersicon esculentum SUT2 and C-terminal halves of StSUT1. However, single halves do not function independently. Our data suggest that NRT2.4, considered a second half of NRT2, complements nitrite uptake activity in Hansenula polymorpha. We do not know whether NRT2.4 plus NRT2.5, or single NRT2.5 are able to reconstitute transport activities with differential characteristics, and so further experiments will be required to answer this point.

The silencing of NRT2.4 in Chlamydomonas strains with artificial micro RNA suggests that NRT2.4 participates in nitrite transport, but its activity is masked when the other efficient nitrite transporter NRT2.1/NAR2 is present. In Chlamydomonas, nitrite transport activity could result from oligomerization of NRT2.5 plus NRT2.4 or, like in Hansenula, only NRT2.4. As indicated above, a nitrite transport system III was proposed in Chlamydomonas [38]. However, the present data suggests NRT2.3, NRT2.4, and perhaps NRT2.5 could participate in this activity.

The expression pattern of NRT2.4 and NRT2.5 shows that these genes express very little and are not nitrogen regulated as NRT2.1, NRT2.2, and NRT2.3 [21,38,43]. However, NRT2.4 and NRT2.5 show a co-regulation under our studied conditions and also in a minus nitrogen medium [47]. This co-regulation could support the idea that NRT2.5 (first half) plus NRT2.4 (second half) might constitute a NRT2 transporter.

Finally, although half-size NRT2 transporters have not been reported, recently a half-size NPF has been identified in rice [48]. It is the OsNRT1.1b, a splicing mRNA product of OsNRT1.1, which encodes a six-transmembrane nitrate transporter corresponding to the first half of OsNRT1.1a. Oocytes expressing OsNRT1.1b accumulate more nitrate, at $0.25 \mathrm{mM}$ nitrate, than when the full size OsNRT1.1a is expressed.

To conclude, this work suggests that Chlamydomonas reinhardtii has half-size NRT2 transporters and that one of them (NRT2.4) is sufficient to work as a nitrite transporter. A complex picture seems to emerge for the nitrate/nitrite transporters, a consequence not only from the different families of transporters, but also of the numbers from each family, and the possibility of generating different protein complexes, i.e., NRT2/NAR2, NRT2 alone, half-size NRT2s.

\section{Experimental Section}

\subsection{Strains and Growth Conditions}

The Chlamydomonas reinhardtii strains used in this work have seen studied previously and correspond to the following types: (1) wild type for nitrate assimilation: 6145c (cc-1691), 21gr (cc-1690), 704 is a WT having a reporter gene pNIA1-ARS [49]; (2) strain JA4 lacks NRT2.2 and NAR1.1, and contains NRT2.1/NAR2 [13,50]; and (3) strain D2R4, which lacks NRT2.1, NRT2.2, NAR2, NIA1, NAR1, and contains the reporter gene $p$ NIA1-ARS [51].

Cells were grown in a minimum medium bubbled with $5 \%(v / v) \mathrm{CO}_{2}$-enriched air, and under continuous light [52]. The nitrogen source used for growth was ammonium chloride (8 $\mathrm{mM})$. After growth, cells were washed out of ammonium and transferred to the medium indicated in the Figures. Nitrite transport was inferred from depletion of nitrite $(100 \mu \mathrm{M})$ from the medium as described [38]. Experiments were done at least by duplicate. The Hansenula polymorpha strains WT and $\Delta$ ynt1 mutant were previously described, as the conditions for growth and assay for nitrite transport activity [40]. 


\subsection{Silencing of NRT2.4}

Artificial miRNA construct pChlamyRNA3-NRT2.4 was prepared following the protocol described [39]. The 90-nt oligonucleotides NRT2.4-amiRNA-Fw and NRT2.4-amiRNA-Rev (Supplementary Table S2) were designed by using the program WMD3 Web MicroRNA Designer [53] and contains a $21 \mathrm{nt}$ amiRNA "TAATATGGTAATGTTAGGCTT" for which its target sequence is at the $3^{\prime} \mathrm{UTR}$ of NRT2.4. The double-stranded DNA oligonucleotide was cloned in SpeI into the vector pChlamyRNA3, under the control of PSAD promoter. The correct orientation of the construct was checked by sequencing, and then used to transform Chlamydomonas strains 704, JA4 and D2R4. Transformation was performed with $500 \mathrm{ng}$ of plasmid DNA by using the glass-beads method [54], and the transformants isolated by paromomycin resistance [55].

\subsection{Expression of CrNRT2.4 in Hansenula Polymorpha}

The ORF corresponding to NRT2.4 cDNA was cloned as a SalI-NRT2.4-SpeI fragment into the yeast expression vector $\mathrm{PYNR}-\mathrm{EX}$ and under the control of the yeast nitrate reductase [56]. The construct pYNR-EX-CrNRT2.4 was confirmed by DNA sequencing and used to transform the high affinity nitrate/nitrite transport mutant $\Delta y n t 1$ ( $\triangle$ ynt1::URA3 leu2). The transformants were obtained in a selection medium without leucine, and then checked for the presence of the construction by colony PCR [56].

\section{4. cDNA Synthesis, Isolation of cDNA for NRT2.4 and NRT2.5 and Quantification of Gene Expression}

Total RNA was isolated according to previously described methods [57]. The first strand cDNA was synthesized from total RNA by using QT primer and the iScript Selected cDNA Synthesis Kit [58] and by following manufacturer instructions.

The isolation of NRT2.4 and NRT2.5 cDNAs was designed considering the translation initiation codon from JGI v2 for primers design. Further versions were also considered [41,42]. See Supplementary Table S2 for the primers used, and Figure 1A for a scheme.

Quantification of NRT2.4 and NRT2.5 transcripts was achieved by real-time PCR on an iCycler MyiQ2 real-time PCR detection system (Bio-Rad) using SsoFastTM EvaGreen Supermix (Bio-Rad). For each gene, the data RNA levels were normalized using the ubiquitin ligase gene as an internal standard and expressed as relative values with respect to this housekeeping gene [59]. See Supplementary Table S2 for the primers used.

Acknowledgments: This work was funded by MINECO (Ministerio de Economía y Competitividad, Spain, Grant No. BFU2011-29338) with support of Fondo Europeo de Desarrollo Regional (FEDER) program, Junta de Andalucía (P08-CVI-04157, BIO-128 and BIO-502), and Plan Propio de la Universidad de Córdoba. Authors thank Maribel Macías for skillful technical assistance. We thank the "Servicio Central de Apoyo a la Investigación" (SCAI, University of Córdoba, Spain) for DNA sequencing.

Author Contributions: A.G. and E.F. designed research; J.J.H., V.C., Z.G., and V.M. performed research and analyzed data; E.F., V.C. and A.G. wrote the paper; J.M.S. contributed to the experiments with Hansenula. All authors discussed the results and commented on the manuscript.

Conflicts of Interest: The authors declare no conflict of interest.

\section{References}

1. Crawford, N.M. Nitrate: Nutrient and signal for plant growth. Plant Cell 1995, 7, 859-868. [CrossRef] [PubMed]

2. Bouguyon, E.; Gojon, A.; Nacry, P. Nitrate sensing and signaling in plants. Semin. Cell Dev. Biol. 2012, 23, 648-654. [CrossRef] [PubMed]

3. Ruffel, S.; Krouk, G.; Coruzzi, G.M. A systems view of responses to nutritional cues in Arabidopsis: Toward a paradigm shift for predictive network modeling. Plant Physiol. 2010, 152, 445-452. [CrossRef] [PubMed]

4. Wang, Y.Y.; Hsu, P.K.; Tsay, Y.F. Uptake, allocation and signaling of nitrate. Trends Plant Sci. 2012, 17, 458-467. [CrossRef] [PubMed] 
5. Krapp, A.; David, L.C.; Chardin, C.; Girin, T.; Marmagne, A.; Leprince, A.S.; Chaillou, S.; Ferrario-Méry, S.; Meyer, C.; Daniel-Vedele, F. Nitrate transport and signalling in Arabidopsis. J. Exp. Bot. 2014, 65, 789-798. [CrossRef] [PubMed]

6. Fernández, E.; Galván, A. Inorganic nitrogen assimilation in Chlamydomonas. J. Exp. Bot. 2007, 58, $2279-2287$. [CrossRef] [PubMed]

7. Fernández, E.; Galván, A. Nitrate assimilation in Chlamydomonas. Eukaryot. Cell 2008, 7, 555-559. [CrossRef] [PubMed]

8. Sanz-Luque, E.; Chamizo-Ampudia, A.; Llamas, A.; Galván, A.; Fernández, E. Understanding nitrate assimilation and its regulation in microalgae. Front. Plant Sci. 2015, 6, 899. [CrossRef] [PubMed]

9. Wang, Y.; Huang, Y.; Wang, J.; Cheng, C.; Huang, W.; Lu, P.; Xu, Y.-N.; Wang, P.; Yan, N.; Shi, Y. Structure of the formate transporter FocA reveals a pentameric aquaporin-like channel. Nature 2009, 462, 467-472. [CrossRef] [PubMed]

10. Jia, W.; Tovell, N.; Clegg, S.; Trimmer, M.; Cole, J. A single channel for nitrate uptake, nitrite export and nitrite uptake by Escherichia coli NarU and a role for NirC in nitrite export and uptake. Biochem. J. 2009, 417, 297-304. [CrossRef] [PubMed]

11. Wang, Y.; Li, W.; Siddiqi, Y.; Symington, V.F.; Kinghorn, J.R.; Unkles, S.E.; Glass, A.D.M. Nitrite transport is mediated by the nitrite-specific high-affinity NitA transporter and by nitrate transporters NrtA, NrtB in Aspergillus nidulans. Fungal Genet. Biol. 2008, 45, 94-102. [CrossRef] [PubMed]

12. Cabrera, E.; González-Montelongo, R.; Giraldez, T.; de la Rosa, D.A.; Siverio, J.M. Molecular components of nitrate and nitrite efflux in yeast. Eukaryot. Cell 2014, 13, 267-278. [CrossRef] [PubMed]

13. Rexach, J.; Fernández, E.; Galván, A. The Chlamydomonas reinhardtii Nar1 gene encodes a chloroplast membrane protein involved in nitrite transport. Plant Cell 2000, 12, 1441-1453. [CrossRef] [PubMed]

14. Mariscal, V.; Moulin, P.; Orsel, M.; Miller, A.J.; Fernández, E.; Galván, A. Differential regulation of the Chlamydomonas Nar1 gene family by carbon and nitrogen. Protist 2006, 157, 421-433. [CrossRef] [PubMed]

15. Wang, Y.; Spalding, M.H. Acclimation to very low $\mathrm{CO}_{2}$ : Contribution of limiting $\mathrm{CO}_{2}$ inducible proteins, LCIB and LCIA, to inorganic carbon uptake in Chlamydomonas reinhardtii. Plant Physiol. 2014, 166, 2040-2050. [CrossRef] [PubMed]

16. Yamano, T.; Sato, E.; Iguchi, H.; Fukuda, Y.; Fukuzawa, H. Characterization of cooperative bicarbonate uptake into chloroplast stroma in the green alga Chlamydomonas reinhardtii. Proc. Natl. Acad. Sci. USA 2015, 112, 7315-7320. [CrossRef] [PubMed]

17. Orsel, M.; Krapp, A.; Daniel-Vedele, F. Analysis of the NRT2 nitrate transporter family in Arabidopsis. Structure and gene expression. Plant Physiol. 2002, 129, 886-896. [CrossRef] [PubMed]

18. Okamoto, M.; Vidmar, J.J.; Glass, A.D.M. Regulation of NRT1 and NRT2 Gene Families of Arabidopsis thaliana: Responses to Nitrate Provision. Plant Cell Physiol. 2003, 44, 304-317. [CrossRef] [PubMed]

19. Unkles, S.E.; Hawker, K.L.; Grieve, C.; Campbell, E.I.; Montague, P.; Kinghorn, J.R. crnA encodes a nitrate transporter in Aspergillus nidulans. Proc. Natl. Acad. Sci. USA 1991, 88, 204-208. [CrossRef] [PubMed]

20. Unkles, S.E.; Zhou, D.; Siddiqi, M.Y.; Kinghorn, J.R.; Glass, A.D.M. Apparent genetic redundancy facilitates ecological plasticity for nitrate transport. EMBO J. 2001, 20, 6246-6255. [CrossRef] [PubMed]

21. Quesada, A.; Galván, A.; Fernández, E. Identification of nitrate transporter genes in Chlamydomonas reinhardtii. Plant J. 1994, 5, 407-419. [CrossRef] [PubMed]

22. Pérez, M.D.; González, C.; Ávila, J.; Brito, N.; Siverio, J.M. The YNT1 gene encoding the nitrate transporter in the yeast Hansenula polymorpha is clustered with genes YNI1 and YNR1 encoding nitrite reductase and nitrate reductase, and its disruption causes inability to grow in nitrate. Biochem. J. 1997, 321, 397-403. [CrossRef] [PubMed]

23. Rowe, J.J.; Ubbink-Kok, T.; Molenaar, D.; Konings, W.N.; Driessen, A.J.M. Nark is a nitrite-extrusion system involved in anaerobic nitrate respiration by Escherichia coli. Mol. Microbiol. 1994, 12, 579-586. [CrossRef] [PubMed]

24. Pao, S.S.; Paulsen, I.T.; Saier, M.H. Major Facilitator Superfamily. Microbiol. Mol. Biol. 1998, 62, 1-34.

25. Reddy, V.S.; Shlykov, M.A.; Castillo, R.; Sun, E.I.; Saier, M.H. The Major Facilitator Superfamily (MFS) Revisited. FEBS J. 2012, 279, 2022-2035. [CrossRef] [PubMed]

26. Forde, B.G. Nitrate transporters in plants: Structure, function and regulation. Biochim. Biophys. Acta 2000, 1465, 219-235. [CrossRef] 
27. Clegg, S.; Feng, Y.; Griffiths, L.; Cole, J.A. The roles of the polytopic membrane proteins NarK, NarU and NirC in Escherichia coli K-12: Two nitrate and three nitrite transporters. Mol. Microbiol. 2002, 44, 143-155. [CrossRef] [PubMed]

28. Zhou, J.J.; Fernández, E.; Galván, A.; Miller, A.J. A high affinity nitrate transport system from Chlamydomonas requires two gene products. FEBS Lett. 2000, 466, 225-227. [CrossRef]

29. Okamoto, M.; Kumar, A.; Li, W.; Wang, Y.; Siddiqi, M.Y.; Crawford, N.M.; Glass, A.D. High-affinity nitrate transport in roots of Arabidopsis depends on expression of the NAR2-like gene AtNRT3.1. Plant Physiol. 2006, 140, 1036-1046. [CrossRef] [PubMed]

30. Orsel, M.; Chopin, F.; Leleu, O.; Smith, S.J.; Krapp, A.; Daniel-Vedele, F.; Miller, A.J. Characterization of a two-component high-affinity nitrate uptake system in Arabidopsis. Physiology and protein-protein interaction. Plant Physiol. 2006, 142, 1304-1317. [CrossRef] [PubMed]

31. Tong, Y.; Zhou, J.J.; Li, Z.; Miller, A.J. A two-component high-affinity nitrate uptake system in barley. Plant J. 2005, 41, 442-450. [CrossRef] [PubMed]

32. Kotur, Z.; Mackenzie, N.; Ramesh, S.; Tyerman, S.D.; Kaiser, B.N.; Glass, A.D.M. Nitrate transport capacity of the Arabidopsis thaliana NRT2 family members and their interactions with AtNAR2.1. New Phytol. 2012, 194, 724-731. [CrossRef] [PubMed]

33. Yong, Z.; Kotur, Z.; Glass, A.D.M. Characterization of an intact two-component high-affinity nitrate transporter from Arabidopsis roots. Plant J. 2010, 63, 739-748. [CrossRef] [PubMed]

34. Galván, A.; Quesada, A.; Fernández, E. Nitrate and nitrate are transported by different specific transport systems and by a bispecific transporter in Chlamydomonas reinhardtii. J. Biol. Chem. 1996, 271, 2088-2092. [CrossRef] [PubMed]

35. Akhtar, N.; Karabika, E.; Kinghorn, J.R.; Glass, A.D.; Unkles, S.E.; Rouch, D.A. High-affinity nitrate/nitrite transporters NrtA and NrtB of Aspergillus nidulans exhibit high specificity and different inhibitor sensitivity. Microbiology 2015, 161, 1435-1446. [CrossRef] [PubMed]

36. Available online: www.tcdb.org (accessed on 25 October 2015).

37. Available online: http://bioinformatics.oxfordjournals.org/content/30/6/884.full.pdf+html (accessed on 27 October 2015).

38. Rexach, J.; Montero, B.; Fernández, E.; Galván, A. Differential regulation of the high affinity nitrite transport systems III and IV in Chlamydomonas reinhardtii. J. Biol. Chem. 1999, 274, 27801-27806. [CrossRef] [PubMed]

39. Molnar, A.; Bassett, A.; Thuenemann, E.; Schwach, F.; Karkare, S.; Ossow-ski, S.; Weigel, D.; Baulcombe, D. Highly specific gene silencing by artificial microRNAs in the unicellular alga Chlamydomonas reinhardtii. Plant J. 2009, 58, 165-174. [CrossRef] [PubMed]

40. Machín, F.; Medina, B.; Navarro, F.J.; Pérez, M.D.; Veenhuis, M.; Tejera, P.; Lorenzo, H.; Lancha, A.; Siverio, J.M. The role of $Y_{n t 1}$ in nitrate and nitrite transport in the yeast Hansenula polymorpha. Yeast 2004, 3, 265-276. [CrossRef] [PubMed]

41. Available online: http://genome.jgi.doe.gov/Chlre4/Chlre4.home.html. (accessed on 23 October 2015).

42. Available online: http://phytozome.jgi.doe.gov/pz/portal.html. (accessed on 27 October 2015).

43. Quesada, A.; Gómez, I.; Fernández, E. Clustering of the nitrite reductase gene and a light-regulated gene with nitrate assimilation loci in Chlamydomonas reinhardtii. Planta 1998, 206, 259-265. [CrossRef] [PubMed]

44. Camargo, A.; Llamas, A.; Schnell, R.A.; Higuera, J.J.; González-Ballester, D.; Lefebvre, P.A.; Fernández, E.; Galván, A. Nitrate signaling by the regulatory gene NIT2 in Chlamydomonas. Plant Cell 2007, 19, 3491-3503. [CrossRef] [PubMed]

45. Navarro, M.T.; Guerra, E.; Fernández, E.; Galván, A. Nitrite reductase mutants as an approach to understanding nitrate assimilation in Chlamydomonas reinhardtii. Plant Physiol. 2000, 122, 283-290. [CrossRef] [PubMed]

46. Reinders, A.; Schulze, W.; Thaminy, S.; Stagljar, I.; Frommer, W.B.; Ward, J.M. Intra- and intermolecular interactions in sucrose transporters at the plasma membrane detected by the split-ubiquitin system and functional assays. Structure 2002, 10, 763-772. [CrossRef]

47. Schmollinger, S.; Mühlhaus, T.; Boyle, N.R.; Blaby, I.K.; Casero, D.; Mettler, T.; Moseley, J.L.; Kropat, J.; Sommer, F.; Strenkert, D.; et al. Nitrogen-Sparing Mechanisms in Chlamydomonas Affect the Transcriptome, the Proteome, and Photosynthetic Metabolism. Plant Cell 2014, 26, 1410-1435. [CrossRef] [PubMed] 
48. Fan, X.; Feng, H.; Tan, Y.; Xu, Y.; Miao, Q.; Xu, G. A putative 6 trans-membrane nitrate transporter OsNRT1.1b plays a key role in rice under low nitrogen. J. Integr Plant Biol. 2015. Available online: www.wileyonlinelibrary.com/journal/jipb (accessed on 25 September 2015). [CrossRef] [PubMed]

49. Higuera, J.J.; Fernández, E.; Galván, A. Chlamydomonas NZF1, a tandem-repeated zinc finger factor involved in nitrate signalling by controlling the regulatory gene NIT2. Plant Cell Environ. 2014, 37, 2139-2160. [CrossRef] [PubMed]

50. Rexach, J.; Llamas, A.; Fernández, E.; Galván, A. The activity of the high-affinity nitrate transport system I (NRT2;1, NAR2) is responsible for the efficient signalling of nitrate assimilation genes in Chlamydomonas reinhardtii. Planta 2002, 215, 606-611. [CrossRef] [PubMed]

51. Llamas, A.; Igeño, M.I.; Galván, A.; Fernández, E. Nitrate signalling on the nitrate reductase gene promoter depends directly on the activity of the nitrate transport systems in Chlamydomonas. Plant J. 2002, 30, 261-271. [CrossRef] [PubMed]

52. Harris, E. The Chlamydomonas Sourcebook; Academic Press: New York, NY, USA, 2009.

53. Available online: http://wmd3.weigelworld.org/cgi- bin/webapp.cgi?page=Home;project=stdwmd (accessed on 25 July 2015).

54. Kindle, K.L. High-frecuency nuclear transformation of Chlamydomonas reinhardtii. Proc. Natl. Acad. Sci. USA 1990, 87, 1228-1232. [CrossRef] [PubMed]

55. González-Ballester, D.; de Montaigu, A.; Higuera, J.J.; Galván, A.; Fernández, E. Functional genomics of the regulation of the nitrate assimilation pathway in Chlamydomonas. Plant Physiol. 2005, 137, 522-533. [CrossRef] [PubMed]

56. Martín, Y.; Navarro, F.J.; Siverio, J.M. Functional characterization of the Arabidopsis thaliana nitrate transporter CHL1 in the yeast Hansenula polymorpha. Plant Mol. Biol. 2008, 68, 215-224. [CrossRef] [PubMed]

57. Sambrook, J.; Fritsch, E.F.; Maniatis, T. Molecular Cloning: A Laboratory Manual, 2nd ed.; Cold Spring Harbor Laboratory Press: New York, NY, USA, 1989.

58. Available online: www.bio-rad.com (accessed on 28 July 2015).

59. González-Ballester, D.; Camargo, A.; Fernández, E. Ammonium transporter genes in Chlamydomonas: The nitrate-specific regulatory gene Nit2 is involved in Amt1;1 expression. Plant Mol. Biol. 2004, 56, 863-878. [CrossRef] [PubMed]

(c) 2016 by the authors; licensee MDPI, Basel, Switzerland. This article is an open access article distributed under the terms and conditions of the Creative Commons by Attribution (CC-BY) license (http:/ / creativecommons.org/licenses/by/4.0/). 